\title{
Analysis of the hydrogen-rich magnetic White Dwarfs in the SDSS
}

\author{
Baybars Külebi $^{1}$, Stefan Jordan ${ }^{1}$, Fabian Euchner ${ }^{2}$, and Heiko Hirsch ${ }^{3}$ \\ ${ }^{1}$ Astronomisches Rechen-Institut, Mönchhofstrasse 12-14, D-69120 Heidelberg, Germany \\ ${ }^{2}$ Swiss Seismological Service, ETH Zürich, Sonneggstrasse 5, CH-8092 Zürich, Switzerland \\ ${ }^{3}$ Dr.-Remeis-Sternwarte, Bamberg, Sternwartestrasse 7, D-96049 Bamberg
}

\begin{abstract}
We have calculated optical spectra of hydrogen-rich (DA) white dwarfs with magnetic field strengths between $1 \mathrm{MG}$ and $1000 \mathrm{MG}$ for temperatures between $7000 \mathrm{~K}$ and $50000 \mathrm{~K}$. Through a least-squares minimization scheme, we have analyzed the spectra of 114 magnetic DAs from the Sloan Digital Survey (SDSS; 95 previously published plus 14 newly discovered within SDSS).
\end{abstract}

Keywords. Stars: white dwarfs - magnetic fields

\section{Introduction}

White dwarfs with magnetic fields between $10^{4}$ and $10^{9} \mathrm{G}$ are thought to represent more than $10 \%$ of the total population of white dwarfs (Liebert et al. 2003). In this work we present the re-analysis of the 95 DA Magnetic White Dwarfs (MWDs) discovered by Schmidt et al. (2003) and Vanlandingham et al. (2005), plus the analysis of 19 additional objects from SDSS up to Data Release 6 (9583 $\mathrm{deg}^{2}$; http://www.sdss.org/dr6/).

Schmidt et al. (2003) and Vanlandingham et al. (2005) determined the field strengths and the inclinations of magnetic dipoles by comparing the observed spectra visually with model spectra calculated using a simplified radiation transfer code (Latter et al. 1987). Their and our results are compared on Fig. 1.

\section{Analysis}

The magnetic field geometry of the MWDs was determined with a modified version of the code developed by Euchner et al. (2002). This code calculates the total flux (and circular polarization) spectra for an arbitrary magnetic field topology by adding up appropriately weighted model spectra for a large number of surface elements. Magnetic field geometries are accounted for by a centered or an offset dipoles in this work. The model parameters are the magnetic dipole field strength $B_{d}$, the effective temperature $T_{\text {eff }}$, the inclination of the dipole axis $i$, and an offset along the magnetic dipole axis $z_{\text {off }}$, if offset dipoles are used. The observed spectra are fitted using an evolutionary algorithm (Rechenberg 1994) with a least-squares quality function and all of our fits resulted in reduced $\chi^{2}$ values between 0.8 and 3.0 except for some high-field objects.

\section{Discussion}

In many cases offset dipole models resulted in significantly better fits than the models with centered dipoles. In particular some MWDs with high field strengths (> $50 \mathrm{MG}$ ), where the spectra become very sensitive to the details of the magnetic field geometry, are not accounted for by our simple models. 
SDSS has nearly tripled the number of MWDs; therefore the completeness of the total known MWD population is strongly affected by the selection biases of SDSS (priority selection of the spectroscopic targeting; Stoughton et al. 2002).

High-field MWDs are thought to be remnants of magnetic Ap and Bp stars. If flux conservation is assumed, population synthesis models predict the majority of MWDs' polar field strengths to be in the interval 50-500 MG (Wickramasinghe \& Ferrario 2005). On the pther hand in our sample objects with magnetic field strengths lower than 50 MG are more numerous than the objects with higher magnetic field strengths (see Fig. 2), partly due to the selection biases. Nevertheless our result is consistent with previous ones and supports the hypothesis that magnetic fossil fields from Ap/Bp stars alone are not sufficient to produce the observed number of MWDs (Wickramasinghe \& Ferrario 2005). Possible progenitor populations are A and B stars with magnetic field strengths below $100 \mathrm{G}$ or magnetic F stars (Schmidt et al. 2003), which both are currently unobserved.

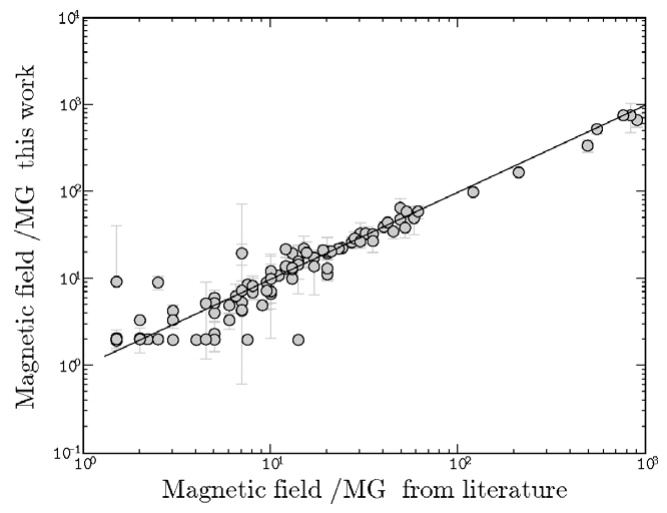

Figure 1. Comparison of dipole magnetic field fit values in this work versus Schmidt et al. (2003), Vanlandingham et al. (2005).

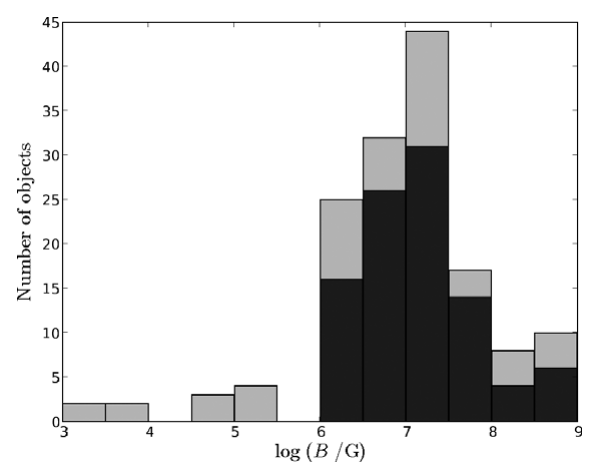

Figure 2. Histogram of magnetic white dwarfs in equal intervals of $\log B$. Gray columns represent the number of all DA MWDs and black shades represent the the contribution of SDSS to DA MWDs.

\section{References}

Euchner, F., Jordan, S., Beuermann, K., Gänsicke, B. T., \& Hessman, F. V. 2002, A 6 A 390, 633

Latter, W. B., Schmidt, G. D., \& Green, R. F. 1987, ApJ 320, 308

Liebert, J., Bergeron, P., \& Holberg, J. B. 2003, AJ 125, 348

Rechenberg, I. 1994, Werkstatt Bionik und Evolutionstechnik No. 1 (Stuttgart: frommannholzboog)

Schmidt, G. D., Harris, H. C., Liebert, J., et al. 2003, ApJ 595, 1101

Stoughton, C., Lupton, R. H., Bernardi, M., et al. 2002, AJ 123, 485

Vanlandingham, K. M., Schmidt, G. D., Eisenstein, D. J., et al. 2005, AJ 130, 734

Wickramasinghe, D. T. \& Ferrario, L. 2005, MNRAS 356, 1576 